\title{
LA TRANSICIÓN DE LA GUERRA A LA PAZ: ANÁLISIS DESDE LA FILOSOFÍA DE LA HISTORIA DE IMMANUEL KANT Y JOHANN GOTTFRIED HERDER ${ }^{1}$
}

The Transition from War to Peace: Analysis from the Philosophy of History of Immanuel Kant and Johann Gottfried Herder ${ }^{1}$

$\frac{\text { Juan Sebastián García-Acevedo }^{2}}{\text { Alejandra Turbay Fontalvo }}$

Fecha de Recepción: Noviembre 4 de 2013

Fecha de Aceptación: Noviembre 11 de 2013

SUMARIO: 1. Introducción; 2. Ideas generales de la relación entre la filosofía de la historia de Kant y Herder; 2.1. La legislación de la filosofía de la historia; 2.2. El antagonismo propio del ser humano; 2.2.1. Naturaleza y libertad: 2.2.2. Legalidad y moralidad; 3. La guerra como representación del estado de naturaleza; 3.1. Hacia la paz perpetua; 4. Conclusiones; 5. Referencias bibliográficas.

\footnotetext{
${ }^{1}$ El presente artículo es resultado parcial del Proyecto de Investigación "Aspectos Morales de la teoría Jurídica y Política” registrado ante el CIB de la Universidad de San Buenaventura, Cartagena; fue desarrollado de manera cooperativa entre investigadores del Grupo de Investigación de la Escuela Latinoamericana de Cooperación y Desarrollo de la Universidad de San Buenaventura y del Grupo de Investigación de Teoría del Derecho y Problemas Jurídicos Contemporáneos de la Universidad de Cartagena.

${ }^{2}$ Docente Investigador de la Facultad de Derecho y Ciencias Políticas de La Universidad de San Buenaventura; Miembro del Grupo de Investigación de la Escuela Latinoamericana de Cooperación y Desarrollo (GIELACID) de la Universidad de San BuenaventuraCartagena. Abogado de la Universidad de Cartagena, Egresado de la Maestría en Filosofía de la Universidad Nacional de Colombia. Juan.Garcia@usbctg.edu.co

${ }^{3}$ Estudiante de X semestre del programa Derecho, de la Facultad de Derecho y Ciencias Políticas de la Universidad de Cartagena. Miembro del Grupo de Investigación de Teoría del Derecho y Problemas Jurídicos Contemporáneos de la Universidad de Cartagena. alejitaturbay@hotmail.com.
} 


\section{COMO SE CITA ESTE ARTÍCULO (APA 6)}

García-Acevedo, J. S., Turbay Fontalvo, A. (2014) La Transición de la guerra a la paz: Análisis desde la Filosofía de la Historia de Immanuel Kant y Johann Gottfried Herder. (Y. Carrillo De la rosa, Ed.) Revista Jurídica Mario Alario D'Filippo, VI (11), pág. 123-145

\section{RESUMEN}

Cuando una nación atraviesa la transición de un estado de guerra a la paz surgen unos cambios a nivel social, político y jurídico. El hombre se ve en la necesidad de crear un mecanismo que impida la repetición de las hostilidades y garantice el mantenimiento de la paz. El período de la llustración aparece como un antecedente importantísimo, en el que la transición del Estado Absolutista al Estado de Derecho hizo que el hombre cambiara toda la tradición jurídica existente e instaurara un nuevo sistema que permitiera el goce efectivo de los derechos adquiridos. Immanuel Kant y Johann Herder desarrollaron sus obras en el contexto de la Ilustración y, a través de la Filosofía de la Historia, crearon un referente importante para el análisis de la guerra como estado de naturaleza y la paz -que debe ser instaurada y protegida por el hombre-como principal logro de la sociedad civil. Este trabajo pretende analizar tales teorías y traerlas al contexto del siglo XXI.

\section{PALABRAS CLAVE}

Immanuel Kant, Johann Gottfried Herder, paz, guerra, transición, filosofía de la historia, estado de naturaleza, moralidad, legalidad, libertad.

\section{ABSTRACT}

When a nation goes through the transition from a state of war to peace some changes to social, political and legal arise. The man is the need to create a mechanism to prevent the recurrence of hostilities and ensure the maintenance of peace. The period of the Enlightenment appears as an important precedent, in which the transition from absolutist state the rule of law caused the man to change all existing legal tradition and establish a new system that would allow the effective enjoyment of acquired rights. Immanuel Kant and Johann Herder developed his works in the context of the Enlightenment and, through the philosophy of history, created an important reference for the analysis of war as a state of nature and peace which must be instituted and protected by the man as the main achievement of civil society. This paper analyzes such theories and bringing them to the context of XXI century.

\section{KEYWORDS}

Immanuel Kant, Johann Gottfried Herder, peace, war, transition, philosophy of history, state of nature, morality, legality, liberty.

\footnotetext{
${ }^{4}$ Película, "Tesis sobre un homicidio" (Argentina-España, 2012) dirigida por Hernán Goldfrid.
} 


\section{INTRODUCCIÓN}

Este trabajo pretende realizar un análisis a partir de los principios de la filosofía kantiana y el historicismo herderiano, en la transición de la guerra a la paz. En primer lugar se plantarán las ideas generales de la relación entre la filosofía de la historia de Kant y Herder, y como sus teorías representaron un hito en la interpretación filosófica de la historia, a pesar de existir una polémica entre la posición de ambos con respecto al período de la llustración. A continuación, explicaremos por qué para Kant el filósofo de la historia es un legislador de la razón, cuya función es dar un orden al aparente caos de la historia (Kant, 1784). Además estudiaremos lo que para Kant constituye un plan oculto de la Naturaleza y cómo el hombre está obligado a cumplirlo. De aquí surgirá el siguiente punto acerca de la insociable sociabilidad de los hombres, como antagonismo propio del ser humano, el cual actúa como detonante de las dicotomías entre naturaleza y libertad, y, moralidad y legalidad.

En cuarto lugar hablaremos de la necesidad de establecer una sociedad civil, como requisito sine qua non para alcanzar la paz. El concepto de estado de naturaleza para Kant, a diferencia de Hobbes, se representa en la guerra y en imposibilidad del hombre de establecer una armonía entre la legalidad y la moralidad. Finalmente trataremos sobre el camino para la paz perpetua y como esta representa el fin del plan oculto de la naturaleza y lleva a hombre a superar el antagonismo en el cual ve la sociabilidad como algo insoportable, pero ineludible.

La presente investigación resulta relevante para el derecho en el contexto colombiano e internacional por lo siguiente. Como consecuencia del proceso de paz que se viene adelantando entre el Gobierno Nacional y las FARC-EP, en la Habana, Cuba; es menester que quienes nos preparamos para un cambio social, político y jurídico, podamos identificar de forma clara la importancia del fin del conflicto como medida eficaz para salir del estado de naturaleza -en términos kantianos- e instaurar una sociedad civil, en la cual se logre la anhelada paz. Kant, como uno de los filósofos más importantes de la modernidad, resulta un punto de referencia idóneo para realizar este análisis (Jaramillo Vélez, 2004), pues para él, el estado de naturaleza se manifiesta en la guerra, en la incapacidad del hombre para establecer una relación armónica entre la legalidad y la moralidad; y es solo mediante una constitución moral, en la cual los defectos del Estado se resuelvan según el derecho y no mediante el uso de la fuerza, que se puede alcanzar esta finalidad. (Gil Olivera, 2004)

Herder, a su vez, fue un historicista que vivió -al igual que Kant- el período de la llustración y sus escritos son útiles para entender el papel de la historia en la preservación de la cultura de un pueblo, luego de un período transicional. De esta forma, Kant y Herder, al vivir el paso del estado absolutista, al estado de derecho, son el referente ideal para entender, a través de la filosofía de historia, la necesidad de los pueblos de evaluar la idoneidad de su sociedad civil y sus instituciones jurídicas, antes de entrar en un período de transición de la guerra, como estado de naturaleza, a la paz.

Esta investigación es de tipo analítica, puesto que se pretende hacer un análisis la transición de la guerra a la paz, desde el punto de vista de la filosofía de Immanuel Kant y el historicismo de Johann 
Herder. Estudiaremos los elementos relacionados con la guerra, como estado de naturaleza, para así comprender la importancia de la instauración de la paz en una sociedad civil. Es una investigación documental y de carácter ius-filosófica.

\section{IDEAS GENERALES DE LA RELACIÓN ENTRE LA FILOSOFÍA DE LA HISTORIA DE KANT Y HERDER}

Lo que el mundo actual conoce por Filosofía de la Historia está íntimamente ligado al pensamiento de Kant, al de Herder y la polémica surgida entre estos pensadores que desarrollaron su obra en la misma época de la historia. Sin embargo, lo que ahora parece un gran avance de la Modernidad, en su momento fue una crítica, por parte de Herder, contra la llustración; lo que representa la primera brecha que diferencia su pensamiento con el de Kant. El libro También una filosofía de Herder plantea muchas críticas en contra del gobierno, el "también" tiene un significado irónico y hace referencia a la proliferación de ensayos que, siguiendo la "moda" del término "Filosofía de la Historia" generada por Voltaire -un filosofo de la llustración-, se publican en la época. (Sisto, 2006) Para Herder, el defecto fundamental de la llustración es su egocentrismo histórico, el cual da lugar a un anacronismo en la consideración de la historia; mientras que para Kant, un filósofo con un pensamiento inagotable y ya famoso en su tiempo, la llustración es necesaria para el futuro de la razón y de la moral, y por esto defendía, con medios limpiosel espíritu ilustrado. (Villacañas, 1999)

La llustración juzga moralmente las épocas pasadas en función de sus propios estándares, se dedica a denigrar siglos enteros acusándolos de barbarie, de miserable derecho público, superstición y necedad; lo cual, para Herder, representa un desconocimiento de la realidad, porque la naturaleza humana se forma progresivamente y los ideales de virtud deben ser deducidos del compendio de su siglo(Herder, 1774). Todo lo contrario a lo propuesto por Kant, para quien el ejercicio de la libertad y la naturaleza humana están ligados al fenómeno de la llustración. Aquí, el hombre hace "uso público de la razón" -uno de los grandes ideales ilustrados- y levanta la voz para considerar las cuestiones que atañen a todos los seres humanos sin excepción (Kant, 1784). "Kant, calladamente pero con gran perseverancia, precisión y sistematicidad, construyó un pensamiento capaz de superar cualquier tentación de olvido por parte de las generaciones futuras". (Mayos, 2004) Herder, causo quizá impacto en su tiempo, es reconocida su importancia en la historia y penetró profundamente en lo humano para historiarlo y comprenderlo, pero en la filosofía no se le reconocen plenamente sus méritos.

La segunda brecha entre el pensamiento de Kant y el de Herder, se basa en la recurrencia a Dios como fundamento o hilo conductor de su obra. Ambos autores plantean que la historia es cognoscible y narrable y lo que predetermina lo humano es una instancia suprahumana, parahumana o protohumana, la cual es previa a lo humano y también más allá, pues es ella la que acabará por definir y crear lo humano mismo; para Herder esta instancia es Dios, para Kant es la naturaleza (Mayos, 2004). Herder, era un cristiano, como muchos en su época, para él la primera creación histórica de la humanidad es el Génesis, una creación básicamente biológico-corporal y es pensada como dada de una vez por todas. Esta debe ser completada por la segunda creación, la cual es la guía histórica de la humanidad, es básicamente espiritual y política y marca el largo proceso de la historia humana, porque en ella viene a devenir y realizarse lo humano en tanto que tal (Mayos, 2004), y dice Herder: 
"Si hay un Dios en la Naturaleza, también lo hay en la historia, porque también el hombre es parte de la creación y debe ajustarse aun [la diferencia del resto de los seres creados ya completos y fijos] en medio de sus más salvajes perversiones y pasiones a las leyes que no son menos hermosas y excelentes que las que rigen todos los cuerpos celestes y humanos." (Mayos, 2004)

La revelación divina, para Herder, no depende solo de la lectura de la Biblia, sino que se estudia a través del mundo, de la historia y de la realidad misma. Por lo que también resalta:

“Quien desee solo especulaciones metafísicas, las tendrá en un camino más corto; mas yo creo que estas, separadas de las experiencias y analogías de la naturaleza, son un viaje por los aires que raras veces llevará a buen puerto." (Sisto, 2006)

Kant, por su parte, se niega a recurrir a Dios, pero utiliza a la Naturaleza como su instancia suprahumana y habla, por principio de un "plan oculto de la Naturaleza" (Kant, 1784) porque esta actúa bajo sus designios y escribe recto, sobre renglones torcidos (Mayos, 2004), si el hombre pretende conocer los designios de la naturaleza, puede hacerlo mediante una idea de la Razón guiada por unas necesidades relacionadas con la teoría del "als ob" o el "como si", en la que, el problema de la libertad afecta solo al hombre, porque somos seres morales y al mismo tiempo seres naturales. El hombre como ser moral es libre y debe actuar como si fuera libre. La moralidad, para Kant, no es un hecho, es una exigencia. La libertad es una exigencia de la Razón y es necesidad de esta sentar un "hilo conductor" que pueda ser a la vez un "hilo propulsor" de los acontecimientos. (Kant, 1784)

Esto último, es casi tan importante para Kant, como para Herder.

\subsection{LA LEGISLACIÓN DE LA FILOSOFÍA DE LA HISTORIA}

"La filosofía es la ciencia de la relación de todos los conocimientos con los fines esenciales de la razón humana y el filósofo es un legislador de esa misma razón, no un artífice de ella." (Kant, 1781)

Con esa frase Kant define lo que es la filosofía y su función en el estudio de la conducta humana. Lo que se pretende al legislar es "alcanzar unos principios que den orden al aparente caos de la historia, sonsacándole un sentido y haciéndola profetizar" (Martínez Ferro, 2001), para Kant, la filosofía de la historia no debe interesarse por relatar hechos de manera descriptiva o enumerando dinastías, reinados y batallas, sino que se debe intentar comprender un orden, un curso regular de los hechos históricos, los llamados "hilos conductores" (Kant, 1784) de la historia. Señala Kant:

“El intento filosófico de elaborar la historia universal del mundo según un plan de la Naturaleza referido a la perfecta unificación civil de la especie humana, se debe considerar como posible y ventajosa para dicha intención natural." (Kant, 1784, pág. 30)

La historia universal para Kant, debe tener un propósito cosmopolita (Kant, 1784), es decir, que además de crear una unidad universal, se rija por leyes de lo que debería ser y no por leyes de lo que 
es, puesto que la historia a futuro se debe regir por leyes de la libertad y no de la naturaleza, al ser la historia de las costumbres humanas su objeto de estudio (Martínez Ferro, 2001). A su vez, si se estudiara sobre el pasado de la historia, las leyes naturales serían la legislación adecuada porque el motivo de estudio recaería en la historia natural de la humanidad.

En principio, para Kant, el hombre crea sus propias reglas e intenciones, estas a su vez, nacen de un instinto natural, dado precisamente por la Naturaleza. Aquí, es preciso preguntarse ¿cómo hace el hombre para crear dichas reglas?, la respuesta dada por Kant, se basa en la facultad otorgada al hombre por la razón. Al ser la única especie dotada de racionalidad, el hombre es el encargado de cumplir el plan de la Naturaleza, pero para lograrlo es necesario, primero, que se desarrolle completamente en la especie y no como individuo; y segundo, que haga libre uso de la razón. En este último punto, Kant entiende que la razón no actúa de forma instintiva, sino que necesita ensayar e instruirse para así sobrepasar la inteligencia de los demás, lo cual no es posible en el soplo de vida de un individuo, por lo que este proceso se dará a través de una serie de generaciones que se transmitan unas a otras la ilustración alcanzada, lo que finalmente constituiría la materialización del plan de la Naturaleza. (Kant, 1784, pág. 19)

Respecto a la discusión sobre si la existencia de la humanidad tiene algún sentido o propósito y si este se realiza en la forma de un desarrollo progresivo y gradual de los pueblos a lo largo del tiempo, Herder discrepa de la idea de Kant sobre la progresión de la humanidad en forma conjunta, pues considera que en primera instancia cada pueblo constituye una manifestación del plan divino (Herder, 1785), valiosa y respetable por sí, y que la ejecución de dicho plan solo se puede entender en la medida en que se capte el espíritu que lo individualiza de otros pueblos (Volksgeist). De esta forma, si bien existe un plan cuya finalidad debe ser perseguida por el hombre, este proviene, según Herder, de la sabiduría y omnipotencia divina y no de un plan oculto de la Naturaleza, como establecia Kant.(Bolívar Espinoza \& Cuellar Saavedra, 2009)

\subsection{EL ANTAGONISMO PROPIO DEL SER HUMANO}

El hombre, para Kant, tiene una propensión a socializarse y también posee una gran inclinación a individualizarse, esta tal resistencia es conocida como la insociable sociabilidad de los hombres, la cual se entiende como la inclinación del hombre a entrar en sociedad y al mismo tiempo una perpetua amenaza de disolverla. Esto responde a que el hombre encuentra en la sociabilidad el progreso adecuado de su condición de hombre y adquiere el sentimiento de desarrollar, según Kant, sus disposiciones naturales; y a su vez, ve en la individualización la posibilidad de poder dirigir todo según su manera de pensar, porque sabe que viviendo en sociedad podría encontrar resistencias por todos lados. De este modo, el hombre se ve en el antagonismo que lo obliga a no poder soportar, pero tampoco evitar, la sociabilidad. A raíz de esto, nacen los primeros rasgos de cultura y se le otorga un valor social al hombre; se desarrollan la ética, los principios y surge la dicotomía entre naturaleza y libertad, y, moralidad y legalidad. 


\subsubsection{Naturaleza y libertad}

Son dos objetos de la filosofía, como legislación de la razón humana. La Naturaleza es todo lo que es y tiene un plan para el desarrollo de todas sus disposiciones, el medio del cual se sirve la Naturaleza para lograr la ejecución de este plan es el antagonismo propio del ser humano. En este orden de ideas, da la impresión, que la Naturaleza usa al hombre como un títere para lograr sus propósitos, sin embargo, según Kant, la Naturaleza se sirve de la insociable sociabilidad humana, para conducir al hombre hacia el ideal del mundo moral. (Martínez Ferro, 2001)

Kant establece que el hombre es un ser esencialmente libre y capaz de darse sus propios fines, empero, esto entra en una aparente contradicción con lo también dicho por Kant respecto a que es la Naturaleza la que dicta el plan cuyo cumplimiento es la finalidad del hombre. No obstante, Kant no sacrifica la libertad humana, por el contrario, tanto la libertad como la Naturaleza se orientan a la realización del hombre como ser moral.

\subsubsection{Legalidad y moralidad}

Si bien estos conceptos aparecen como contradictorios, en realidad son inseparables. La moralidad posibilita la autonomía de los individuos y permite la salida del hombre de su minoría de edad, de la cual él mismo es culpable (Kant, 1784), pero lo más importante es que la moralidad condiciona la aparición de la legalidad, es decir, la legalidad solo es posible si y solo si se apoya en la moralidad. De esta forma, un sistema legal obliga al hombre a cumplir la ley aunque él no sea un hombre moral, pero respeta un sistema, que para Kant, si lo es (Martínez Ferro, 2001). La legalidad y la moralidad son dos progresos humanos cuyo principal fin es obligar al hombre a salir del estado de naturaleza y evitar la guerra.

\section{LA GUERRA COMO REPRESENTACIÓN DEL ESTADO DE NATURALEZA}

"El estado de naturaleza es más bien la guerra, es decir, un estado donde si bien las hostilidades no se han declarado, si existe una constante amenaza." (Kant, 2003)

A diferencia de autores como Hobbes o Locke, para Kant el Estado de Naturaleza no es un estado en el que hombre está sujeto a la agresión, y a la vida sin ley ni sociedad (García-Acevedo, 2010); en su lugar lo define como la ausencia de paz y la imposibilidad de establecer una armonía entre la moralidad y la legalidad. Para Kant, la guerra la ocasiona cuando las exageraciones de la libertad de uno, comienzan a dañar la libertad de los demás. (Tapias Mejía, 2006)

Para la razón, es necesario abandonar el estado de naturaleza con el fin de constituir la sociedad civil, mediante el contrato originario. Por lo tanto, la paz es un deber ético, comprensible y aceptable (Gil Olivera, 2004), ya que comprendemos que es necesario el ideal común de una vida pacífica, pues la vida es un derecho de todos los seres humanos. Sin embargo, traducir de la práctica a la realidad, resulta de difícil aceptación (Catanzaro, 2010), como ocurre con todos los principios éticos. En la práctica, el derecho no es imperante ni absoluto, son otros los principios de carácter empírico los que ejercen autoridad; principios que nada tienen que ver con la ética (García- 
Acevedo, 2010). Quien tiene el poder en sus manos, no está dispuesto a cederlo y debe ejercer su autoridad de forma violenta y forzada, por lo que el estado jurídico (Gil Olivera, 2004) no es producto de un acuerdo común, sino de una coacción ejercida por los dueños del poder. Plantea Kant:

\begin{abstract}
“En la constitución republicana no puede por menos de ser necesario el consentimiento de los ciudadanos para declarar la guerra. Nada más natural, por tanto, que, ya que ellos han de sufrir los males de la guerra [...] lo piensen mucho y vacilen antes de decidirse a tan arriesgado juego. En cambio en una constitución en la cual el súbdito no es ciudadano, en una constitución no republicana, la guerra es la cosa más sencilla del mundo. El jefe del estado no es un conciudadano sino un amo, y la guerra no perturba en lo más mínimo su vida regalada [...]. La guerra, para él, es una especie de diversión y puede declararla por levísimos motivos, encargando luego al cuerpo diplomático para que cubra las apariencias y rebusque una justificación plausible." (Kant, 2003)
\end{abstract}

Lo anterior, prácticamente aceptado por la gran parte de los filósofos modernos, ha creado una brecha entre ética y política (García-Acevedo, 2010). Los intereses nacionales, las políticas económicas, las razones patrióticas o personales, constituyen los argumentos usados por los gobernantes para justificar la guerra "en efecto, la guerra se hace siempre en defensa de instituciones, pueblos, naciones, ideas o intereses mezquinos y nunca en defensa de los individuos concretos" (Gil Olivera, 2004). Se demuestra entonces que el motivo de la guerra está influenciado por una moralidad utilitarista -completamente opuesta a la moralidad kantiana- en la cual intereses individualistas que no defienden los intereses de la colectividad y pasa por encima de las atrocidades y costos producto de la guerra.

\title{
3.1. HACIA LA PAZ PERPETUA
}

Kant considera que el problema político es el problema crucial de la especie humana (Kant, 2003). En una totalidad cosmopolita, la paz debe procurarse tanto al interior de cada estado como en el ámbito externo. Para Kant, la paz entre los hombres que viven juntos no es un estado de naturaleza, por tanto "la paz debe ser instaurada" (Tapias Mejía, 2006). Al respecto Kant señala:

"La Constitución cuyo fundamentos sean los tres siguientes: 1. Principio de la libertad de los miembros de una sociedad -como hombres-; 2. Principio de la dependencia en que todos se hallan de una única legislación común -como súbditos-; 3. Principio de la igualdad de todos -como ciudadanos-, es la única constitución que nace de la idea del contrato originario, sobre el cual ha de fundarse toda la legislación de un pueblo."(Kant, 2003)

La paz perpetua es una construcción del hombre con la ayuda del hombre, el cual pasa de vivir en una constante guerra para entrar en una sociedad civil (Kant, 1784). La sociedad civil solo se garantiza por medio de una constitución, la cual impediría un eventual abuso y le otorga al hombre el estatus de ciudadano de derecho. 


\section{CONCLUSIONES}

Al realizar esta investigación pudimos concluir que Para Kant, es una tarea imprescindible, necesaria y urgente la realización de la paz perpetua, y por tanto, se hace necesario el sometimiento de los estados a un derecho internacional común de carácter universal, ya que al ser la paz-como fin del antagonismo propio del ser humano- quien nos sacó de la guerra como estado de naturaleza, necesitamos una federación de todos los pueblos de la Tierra, para garantizarla. (Kant, 2003)

En últimas, la naturaleza busca librar al hombre de sí mismo, superando el antagonismo de la insociable sociabilidad del ser, y luego la moralidad conduce al hombre a formar parte de la sociedad civil, para así garantizar la salida del estado de naturaleza y el mantenimiento de la legalidad. Estamos legal y moralmente obligados, en términos kantianos, a alcanzar una paz perpetua dentro de una ciudadanía cosmopolita.

\section{REFERENCIAS BIBLIOGRÁFICAS}

BOLÍVAR ESPINOZA, A., \& Cuellar Saavedra, O. (2009) "J.G. Herder, Filósofo de la Historia, Reaccionario e Innovador". México D.F.: Universidad Autónoma Metropolitana.

CATANZARO, G. (Marzo de 2010) "Derroteros de la Filosofía de la Historia de Herder a Hegel". Buenos Aires: Instituto de Investigaciones Gino Germani - Universidad de Buenos Aires.

GARCíA-ACEVEDO, J. S. (2010) "Ética y Alteridad en los inicios de la Teoría Política de la Modernidad". Razón Práctica Y Asuntos Públicos(15), 20 36.GIL OLIVERA, N. A. (2004) "La paz perpetua o el valor de vivir en paz". En H. Ortiz Rivas, Kant y la Paz Perpetua (págs. 19-24). Bogotá: Ediciones Juridicas Gustavo Ibañez.

HERDER, J. G. (1774) "También una filosofía".

HERDER, J. G. (1785) "Ideas para una filosofía de la historia de la humanidad". JARAMILLO VÉLEZ, R. (2004) "Antecedentes de La Paz Perpetua". En H. Ortíz Rivas, Kant y la Paz Perpetua (págs. 25-41). Bogotá: Ediciones Jurídicas Gustavo Ibáñez.

KANT, I. (1781) "Crítica de la Razón Pura".

KANT, I. (1784) "Idea de una historia universal con propósito cosmopolita". En I. Kant, En defensa de la llustración. Barcelona: Alba Editorial.

KANT, I. (1784) "La respuesta a la pregunta ¿Qué es la llustración?" En I. Kant, En defensa de la llustración. Alba Editorial.

\footnotetext{
${ }^{5}$ Para indagar más a detalle de esta teoría y de esta forma de concebir el derecho en clave de filosofía jurídica, consultar a: García, A. (1998). "Principios y positivismo jurídico." El no positivismo Principialista en las teorías de Ronald Dwokin y Robert Alexy. Centro de estudios políticos y constitucionales, Madrid, España.
} 
KANT, I. (1785) "Recensión de las Ideas para la filosofía de la historia de la humanidad de Johann Gottfried Herder". En I. Kant, En defensa de la Ilustración. Barcelona: Alba Editorial.

KANT, I. (1786) "Comienzo presunto de la historia humana". En I. Kant, En defensa de la Ilustración. Barcelona: Alba Editorial.KANT, I. (1976) "Principios metafísicos del Derecho". Puebla: Cajica.

KANT, I. (1999) "En defensa de la llustración". (M. Cabot, Ed.) Barcelona: Alba Editorial.

KANT, I. (2003) "Fundamentacion de la metafisica de las costumbres. Critica de la razon practica. La paz perpetua". México: Porrua.

KANT, I. (2008) "Los progresos de la metafísica" (bilingüe ed.). (m. Caimi, Trad.) México: Fondo de Cultura Económica. UAM. UNAM.MARTÍNEZ FERRO, H. (2001) "El lugar de la Filosofía de la Historia en el Sistema Kantiano". Conceptos(1), 5-16.MAYOS, G. (2004) "Ilustración frente a Romanticismo. La polémica Kant - Herder en filosofía de la historia". Barcelona: Editorial Herder.

ORTíz RIVAS, H. (2004) "Breve reflexión sobre La Paz Perpetua" de Kant en su bicentenario. En H. Ortíz Rivas, Kant y la Paz Perpetua (págs. 42-60). Bogotá: Ediciones Jurídicas Gustavo Ibáñez.

ORTíZ RIVAS, H. (2004) "Kant y la Paz Perpetua". Bogotá: Ediciones Jurídicas Gustavo Ibáñez.

RODRíGUeZ BALVUENA, M. G. (2004) "Con motivo de los doscientos años de la muerte de Kant: "la Paz Perpetua, un esbozo filosófico". En H. Ortíz Rivas, Kant y la Paz Perpetua (págs. 61-121). Bogotá: Ediciones Jurídicas Gustavo Ibáñez.

SISTO, M. (2006) "El aporte de Herder en 1774, y sus ideas (1784-1791)". En D. Brauer, La Historia desde la Teoría. Buenos Aires: Prometeo Libros.

TAPIAS MEJÍA, A. E. (2006) "Reflexiones en torno a la Filosofia de la Historia de Immanuel Kant". Cartagena: Universidad de Cartagena.

VILLACAÑAS, J. L. (1999) "Crítica y presente: sobre las bases de la llustración kantiana". En I. Kant, En defensa de la llustración. Barcelona: Alba Editorial. 